\title{
Depinning transition of dislocation assemblies: Pileups and low-angle grain boundaries
}

\author{
Paolo Moretti \\ Dipartimento di Fisica Università “La Sapienza," Piazzale A. Moro 2, 00185 Roma, Italy \\ and Center for Materials Science and Engineering, University of Edinburgh, King's Buildings, Kenneth Denbigh Building, \\ Edinburgh EH93JL, United Kingdom \\ M.-Carmen Miguel \\ Departament de Física Fonamental, Facultat de Física, Universitat de Barcelona, Avenida Diagonal 647, E-08028 Barcelona, Spain
}

Michael Zaiser

Center for Materials Science and Engineering, University of Edinburgh, King's Buildings, Sanderson Building, Edinburgh EH93JL, United Kingdom

Stefano Zapperi

INFM UdR Roma 1 and SMC, Dipartimento di Fisica, Università "La Sapienza," Piazzale A. Moro 2, 00185 Roma, Italy

(Received 16 December 2003; revised manuscript received 19 March 2004; published 8 June 2004)

\begin{abstract}
We investigate the depinning transition occurring in dislocation assemblies. In particular, we consider the cases of regularly spaced pileups and low-angle grain boundaries interacting with a disordered stress landscape provided by solute atoms, or by other immobile dislocations present in nonactive slip systems. Using linear elasticity, we compute the stress originated by small deformations of these assemblies and the corresponding energy cost in two and three dimensions. Contrary to the case of isolated dislocation lines, which are usually approximated as elastic strings with an effective line tension, the deformations of a dislocation assembly cannot be described by local elastic interactions with a constant tension or stiffness. A nonlocal elastic kernel results as a consequence of long-range interactions between dislocations. In light of this result, we revise statistical depinning theories of dislocation assemblies and compare the theoretical results with numerical simulations and experimental data.
\end{abstract}

DOI: $10.1103 /$ PhysRevB.69.214103

PACS number(s): 61.72.Lk, 61.72.Mm, 62.20.Fe, 64.60.Fr

\section{INTRODUCTION}

The depinning transition of individual dislocations gliding on their slip plane has been widely investigated in the past ${ }^{1-6}$ in order to explain solid solution hardening, ${ }^{7-9}$ that is, the increase of the yield stress value when solute atoms are present in a crystal. The presence of solute atoms changes the local properties of the host material, resulting in a pinning force on nearby dislocations. ${ }^{8,9}$ This is not the only source of pinning, which can also be provided by particle inclusions or by dislocations in other slip systems. ${ }^{7}$ Several approximate calculations have been performed in the past to obtain the depinning stress from a statistical summation of individual pinning forces. In this respect, collective pinning theories have been very successful in the case of diffuse weak pinning forces, ${ }^{10-12}$ whereas the theory introduced by Friedel $^{13}$ is appropriate in the case of localized strong pinning centers.

From the purely theoretical point of view, dislocations provide a concrete example of a more general problem: that of driven elastic manifolds in quenched random media. ${ }^{14}$ Apart from dislocations, other examples of this general problem are domain walls in ferromagnets, ${ }^{15,16}$ flux lines in type II superconductors, ${ }^{17,18}$ contact lines, ${ }^{19,20}$ and crack fronts. ${ }^{21,22}$ In recent years, a vast theoretical effort has been devoted to understand the depinning transition as a nonequilibrium critical phenomenon. ${ }^{14,23-28}$ The morphology of a depinning line is generally found to be self-affine and can be characterized by a roughness exponent. Other scaling exponents have been introduced to characterize the behavior of correlation lengths and times, the velocity above depinning, and the avalanching motion observed as the critical threshold is approached. Quantitative predictions of the critical exponents have been obtained analytically by the renormalization group, ${ }^{14,23-28}$ and have been confirmed by numerical simulations. $^{24,29-35}$ In the course of time, a deeper level of description and understanding of this phenomenon has been achieved, going far beyond a mere estimate of the depinning force, which has typically been the original motivation to address the problem.

The analysis of the depinning transition in dislocation theory has often been made in the line-tension approximation $^{1-5}$ where dislocations are considered as flexible strings with local elasticity. This analogy is not fully accurate. In fact, the bending of a dislocation produces longrange stress and strain fields ${ }^{36,37}$ and therefore the energy of a dislocation line segment depends on the overall configuration of the dislocation line. As in the case of vortex lines in high-temperature superconductors ${ }^{38}$ or dislocation lines in vortex crystals, ${ }^{39}$ this leads to a logarithmic wave-vector dependence of the effective line tension. This wave-vector dependence does not affect the main features of the depinning transition, ${ }^{6}$ although numerical simulations indicate a slight change in the roughness exponent which is not completely understood. ${ }^{6,34}$ In passing, we note that a surface-tension approximation has also been used to describe the so-called Ze- 


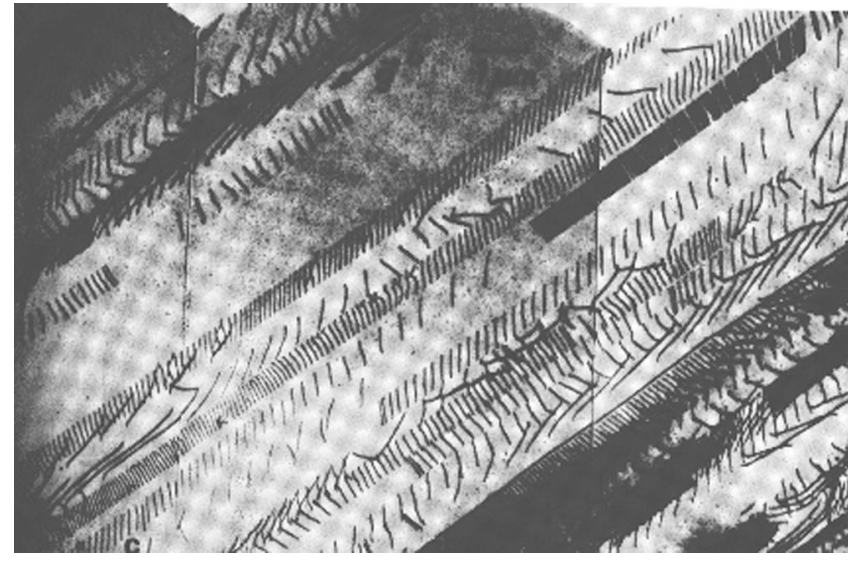

FIG. 1. Transmission electron micrograph taken from a $\mathrm{Cu}-14.4$ at \% $\mathrm{Al}$ single crystal deformed at room temperature; the image shows large regularly spaced dislocation pileups. Courtesy of Plessing and Neuhäuser (Ref. 44).

ner pinning of grain boundaries ${ }^{40,41}$ and its impact on grain growth. $^{42}$

While the behavior of an isolated dislocation pushed through a random distribution of obstacles is at present quite well understood, the results do not necessarily carry over to the more realistic case of collective dislocation motion. Dislocations interact via their long-range stress fields, which may induce intriguing jamming and avalanche like phenomena even in the absence of immobile obstacles. ${ }^{43}$ In most cases, one cannot simply neglect interactions and treat dislocations as isolated objects. Developing an analytical theory of the depinning transition of interacting dislocation lines and/or loops of generic orientations and Burgers vectors in a random solute distribution may be a formidable task. In several instances, however, dislocations are arranged into regular structures that are amenable to analytical treatment. In particular, here we analyze the depinning transition of onedimensional dislocation arrays, viz, regularly spaced pileups and low-angle grain boundaries (LAGB). These relatively simple structures are sometimes observed experimentally (see Fig. 1) and provide a nice illustration of the effect of dislocation interactions on the depinning transition. An early analysis of the depinning of a dislocation pileup was presented in Ref. 45, considering explicitly the emission of dislocations from a source.

We address the problem by first computing, in Sec. II of the present paper, the stress and elastic energy associated with a small deformation of the dislocation arrangement. It turns out that local elasticity approximations are inadequate for dislocation arrays since long-range interdislocation interactions make pileups and low-angle grain boundaries much stiffer than isolated dislocations. The elastic energy is then used in Sec. III to estimate the depinning stress within the framework of statistical pinning theories, using collective pinning theory and Friedel statistics for the weak and strong pinning limits, respectively.

In Sec. IV we investigate the dynamics of dislocation arrays. At stresses close to the depinning stress, the dynamics exhibits critical behavior which can be characterized in terms of scaling exponents. Using previous renormalization-group results, we gain a complete quantitative picture of the depinning transition. In the elastic approximation, pileups and low-angle grain boundaries are equivalent to a standard interface depinning problem with long-range elasticity. In two dimensions (2D), the problem can be mapped to a contact line or to a planar crack, which have been extensively studied in the literature. In three dimensions, the self-stress is similar to the dipolar force in magnetic domain walls and leads to logarithmically rough deformations. In more technical terms, $d=3$ is the upper critical dimension for the transition, which is well described, up to logarithmic corrections, by mean-field exponents.

The scaling exponents associated with the depinning transition describe not only the morphology of the dislocation array but also its dynamics. In order to confirm the validity of the elastic calculations, we perform a series of numerical simulations for a dislocation pileup. We consider a twodimensional system, neglecting the deformation of single dislocations, which amounts to an effective one-dimensional particle model. Simulations of the model display results in agreement with the theory and allow to illustrate some interesting dynamical effects. In particular, the pileup displays a zero-temperature power-law creep relaxation which can be interpreted by scaling relations. Below threshold, the powerlaw relaxation terminates into a pinned configuration, while above threshold there is a crossover to linear creep or average constant velocity sliding. As is common for this class of systems, the motion of the pileup takes place in the form of avalanches whose distribution again can be characterized by scaling exponents.

\section{ELASTICITY}

Developing a theory for collective dislocation depinning requires the basic knowledge of the elastic properties of the dislocation assembly in the first place. In this section, we determine the elastic response of two particular dislocation assemblies: a regularly spaced pileup and a low-angle grain boundary of edge dislocation lines. The two structures are quite similar geometrically; both are one-dimensional arrays of $N$ dislocation lines with the same Burgers vector $\mathbf{b}$ and average line direction $\hat{e}$ (for edge dislocations $\hat{e} \perp \mathbf{b}$ ), but they differ in the relative orientation of the Burgers vector and the array direction $\hat{d}$. In particular, in a pileup a set of edge dislocations lies in the same slip plane (defined by the dislocation line direction $\hat{e}$ and the Burgers vector) so that $\hat{d} \| \hat{b}$ (see Fig. 2 for a particular example with $\hat{e}=\hat{z}$ and $\hat{d}\|\hat{b}\| \hat{y}$ ), whereas in the LAGB the edge dislocations are stacked in the perpendicular plane such that $\hat{d} \perp \hat{b}$ (see Fig. 3 for a particular geometry). We neglect climb, i.e., the motion of a dislocation perpendicular to its slip plane; hence deformations of the structure can occur solely in the direction of $\hat{b}$ both for the pileup and for the LAGB. In this section we derive the shear stress and the elastic energy associated with small deformations of these dislocation assemblies. This is needed in order to derive the yield stress from statistical pinning theories. For completeness, we consider the problem both in two and in three dimensions. 


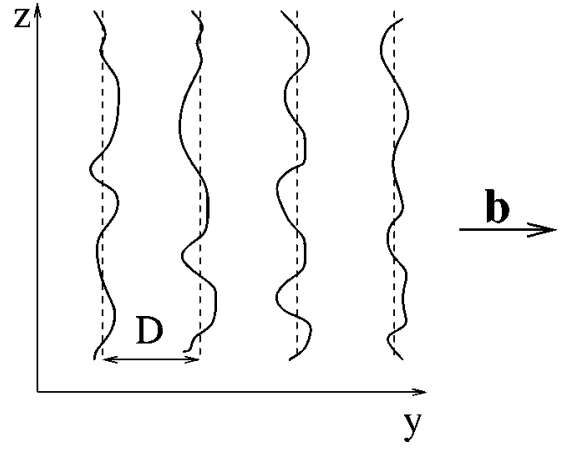

FIG. 2. A regularly spaced dislocation pileup with Burgers vector along the $y$ axis. The ideal configuration is plotted with straight dashed lines, whereas the solid lines represent their possible glide deformations within the slip plane $y z$.

\section{A. Two dimensions}

A two-dimensional model is obtained if we treat the dislocations as rigid lines. In this case, deformations of the dislocation arrangement result only from variations in the position of the dislocations within the one-dimensional arrays they form. We consider the case of a LAGB and then directly extend the result to the pileup. In fact, in linear approximation the elastic energy turns out to be the same in both cases.

Here and throughout the paper, we consider an ideal LAGB as an infinite set of equally spaced edge dislocations lying on the $y z$ plane (without loss of generality we consider the plane $x=0$ ) with Burgers vector pointing along the positive $x$ axis $\mathbf{b}=b \hat{x}$ (see Fig. 3). In the rigid dislocation approximation, each dislocation is described by the coordinates $\left(x_{n}, y_{n}\right)$, where $y_{n}=n D, D$ is the dislocation spacing in the LAGB, and $x_{n}$ is a small displacement out of the $x=0$ plane. The shear stress at the point $(x, y)$ due to a dislocation at $\left(x_{n}, y_{n}\right)$ is given by ${ }^{13,46}$

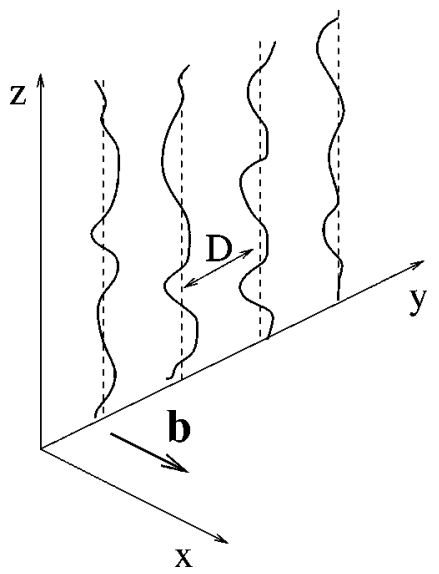

FIG. 3. A regularly spaced low-angle grain boundary where the dislocations' Burgers vector is parallel to the $x$ axis. The ideal configuration is plotted with straight dashed lines in the plane $y z$, whereas the solid lines represent their possible glide deformations within the slip plane $x z$.

$$
\sigma_{x y}^{n}(x, y)=\frac{\mu b}{2 \pi(1-\nu)} \frac{\left(x-x_{n}\right)\left[\left(x-x_{n}\right)^{2}-\left(y-y_{n}\right)^{2}\right]}{\left[\left(x-x_{n}\right)^{2}+\left(y-y_{n}\right)^{2}\right]^{2}},
$$

where $\mu$ is the shear modulus and $\nu$ is the Poisson ratio. The glide component of the total force per unit length on another dislocation $m$ in the LAGB can be readily obtained from the Peach-Koehler expression $\mathbf{f}=(\sigma \cdot \mathbf{b}) \times \hat{e}$ Refs. 13 and 46

$$
f_{x}\left(x_{m}, y_{m}\right)=b \sum_{n=-\infty}^{+\infty} \sigma_{x y}^{n}\left(x_{m}, y_{m}\right)
$$

For small deformations $\left|x_{m}-x_{n}\right| \ll D|m-n|$ we have

$$
f_{x}\left(x_{m}, y_{m}\right)=-\frac{\mu b^{2}}{2 \pi(1-\nu)} \sum_{n=-\infty}^{+\infty} \frac{x_{m}-x_{n}}{\left(y_{m}-y_{n}\right)^{2}},
$$

which can be used to obtain the elastic energy

$$
\begin{aligned}
E & =-\sum_{m=-\infty}^{+\infty} \int f_{x}\left(x_{m}, y_{m}\right) d x_{m} \\
& =\frac{\mu b^{2}}{8 \pi(1-\nu)} \sum_{m=-\infty}^{+\infty} \sum_{n=-\infty}^{+\infty} \frac{\left(x_{m}-x_{n}\right)^{2}}{(m-n)^{2} D^{2}} \quad \text { with } m \neq n .
\end{aligned}
$$

It is instructive to express the elastic energy in Fourier space, where one can easily identify the energy cost of the different modes. For an infinitely long LAGB $N \rightarrow \infty$, we can write the dislocation displacements as

$$
x_{m}=\int_{B Z} \frac{d k}{2 \pi} e^{-i k D m} x(k),
$$

where because of the periodic dislocation arrangement the integral is restricted to the first Brillouin zone (BZ) of the reciprocal space $(-\pi / D<k<\pi / D)$. Using

$$
\sum_{d=1}^{+\infty} \frac{1}{d^{2}}=\frac{\pi^{2}}{6}, \quad \sum_{d=1}^{+\infty} \frac{\cos (\gamma d)}{d^{2}}=\frac{\pi^{2}}{6}-\frac{\pi|\gamma|}{2}+\frac{\gamma^{2}}{4}
$$

we obtain ${ }^{47}$

$$
E=\frac{\mu b^{2}}{8 \pi(1-\nu) D^{2}} \int_{B Z} \frac{d k}{2 \pi}\left(2 \pi|k|-D k^{2}\right) \widetilde{x}(k) \widetilde{x}(-k) .
$$

From this expression, one can see that the elastic interaction kernel $\left(2 \pi|k|-D k^{2}\right)$ is not quadratic in the wave vector, as would be the case for a local elastic line with a constant tension or stiffness, but grows roughly as $|k|$ for long wavelength deformations. This is a consequence of long-range interactions between dislocations in the LAGB which render a much stiffer structure. In the following sections we will explore the consequences of this result in view of the collective pinning of such dislocation structures, something that has been disregarded in previous studies of dislocation depinning.

The elastic energy associated with perturbations of a regularly spaced dislocation pileup can be obtained in an analogous manner. According to the geometric conditions assumed here, all Burgers vectors are now oriented along the positive $y$ axis, and since the dislocations are all in the same slip plane we can now write $x=x_{n}=0$. Proceeding as before, 
the total Peach-Koehler force on dislocation $m$ along the new glide direction is given by

$$
f_{y}\left(0, y_{m}\right)=\frac{\mu b^{2}}{2 \pi(1-\nu)} \sum_{n=-\infty}^{+\infty} \frac{1}{y_{m}-y_{n}} .
$$

Note that the Peach-Koehler forces are now repulsive; however, the stability of the system is ensured in the case of an infinite pileup where the dislocations located at the extremes $($ at $\pm \infty)$ have fixed positions, or for a finite pileup with periodic boundary conditions. Thus, one can also compute the elastic energy cost of small displacements $\delta y_{m}$ of the dislocations in the pileup with respect to their stable positions. Up to first order in $\delta y_{m}$, we obtain a restoring elastic force

$$
f_{y}\left(0, \delta y_{m}\right)=-\frac{\mu b^{2}}{2 \pi(1-\nu)} \sum_{n=-\infty}^{+\infty} \frac{\delta y_{m}-\delta y_{n}}{\left(y_{m}-y_{n}\right)^{2}}
$$

equivalent to the one obtained for the case of the LAGB. The corresponding elastic energy cost is given by Eq. (7) with $x(k)$ replaced by $\delta y(k)$

As a partial conclusion of this section, we emphasize that long wavelength distortions of low-angle grain boundaries and equally spaced pileups of straight dislocation lines with translational invariance along the dislocation axis have the same nonlocal elastic properties, with eigenvalues that grow linearly with the modulus of the wave vector considered.

\section{B. Three dimensions}

In this section, we consider the more general and realistic case of deformable dislocation lines. As before, we consider first the case of a LAGB with Burgers vectors oriented along the $x$ axis, in which each dislocation is now described by a set of coordinates $\left[x_{n}(z), y_{n}, z\right]$. Again, $y_{n}=n D$, but now the displacement $x_{n}(z)$ of the infinitesimal dislocation segment under consideration depends on its position $z$ along the dislocation line (see Fig. 3). The elastic stress field due to a general dislocation line or loop can be obtained, for instance, by considering the line as being composed of elementary segments of infinitesimal length. ${ }^{46}$ Depending on the relative orientation of the Burgers vector and the local tangent vector $\hat{\tau}(z)$, each segment can either have edge $[\hat{\tau}(z) \perp \mathbf{b}]$ or screw character $[\hat{\tau}(z) \| \mathbf{b}]$, or it can be a combination of both. A first approximation of a general dislocation line can be its representation in terms of a succession of only edge and screw segments. ${ }^{48}$ The mathematical form of the elastic stress fields generated by these two types of elementary segments is simple and renders amenable the analytic treatment of the problem. The shear stress created at the point $(x, y, z)$ by an edge dislocation segment of Burgers vector $\hat{b}=b \hat{x}$ and length $\Delta z^{\prime}$ located at $\left(x^{\prime}, y^{\prime}, z^{\prime}\right)$ is given by ${ }^{46}$

$$
\sigma_{x y}(x, y, z)=\frac{\mu b}{4 \pi(1-\nu)} \frac{x-x^{\prime}}{R_{0}^{3}}\left[1-3 \frac{\left(y-y^{\prime}\right)^{2}}{R_{0}^{2}}\right] \Delta z^{\prime},
$$

where

$$
R_{0}^{2}=\left(x-x^{\prime}\right)^{2}+\left(y-y^{\prime}\right)^{2}+\left(z-z^{\prime}\right)^{2},
$$

and the shear stress field due to a screw segment of length $\Delta x^{\prime}$ is $^{46}$

$$
\sigma_{x y}(x, y, z)=-\frac{\mu b}{4 \pi} \frac{z-z^{\prime}}{R_{0}^{3}} \Delta x^{\prime} .
$$

Equations (10) and (12) allow us to calculate the glide component of the total Peach-Koehler force $\mathbf{f}=(\sigma \cdot \mathbf{b}) \times \hat{\tau}$ on an edge or a screw segment. The glide force on an edge segment at $\left[x_{m}(z), y_{m}, z\right]$ has two contributions $f_{x}^{E E}$ and $f_{x}^{S E}$ arising from its respective interactions with other edge or screw segments,

$$
\begin{aligned}
f_{x}^{E E}\left(x_{m}(z), y_{m}, z\right)= & \frac{\mu b^{2}}{4 \pi(1-\nu)} \frac{x_{m}(z)-x_{n}\left(z^{\prime}\right)}{R_{m n}^{3}\left(z, z^{\prime}\right)} \\
& \times\left[1-3 \frac{\left(y_{m}-y_{n}\right)^{2}}{R_{m n}^{2}\left(z, z^{\prime}\right)}\right] \Delta z^{\prime} \Delta z, \\
f_{x}^{S E}\left(x_{m}(z), y_{m}, z\right)= & -\frac{\mu b^{2}}{4 \pi} \frac{z-z^{\prime}}{R_{m n}^{3}\left(z, z^{\prime}\right)} \frac{\partial x_{n}\left(z^{\prime}\right)}{\partial z^{\prime}} \Delta z^{\prime} \Delta z .
\end{aligned}
$$

Note that up to first order in the small displacements $\left[x_{m}(z)\right.$ $\left.-x_{n}\left(z^{\prime}\right) \simeq 0\right]$, the relative distance among segments can be written as $R_{m n}^{2}\left(z, z^{\prime}\right)=\left(y_{m}-y_{n}\right)^{2}+\left(z-z^{\prime}\right)^{2}$. On the other hand, from the general expression for the Peach-Koehler Force written above, it is straightforward to verify that there are no glide forces acting upon any screw segment on the dislocation line. After summing up all nonvanishing contributions, we can obtain the elastic energy as for the two-dimensional case [see Eq. (4)]. The elastic energy can be expressed as the sum $E=E^{E E}+E^{S E}$ of the interaction energies between edgeedge and edge-screw segments. These are given by

$$
\begin{aligned}
& E^{E E}=-\frac{\mu b^{2}}{32 \pi(1-\nu)} \sum_{m, n} \iint d z d z^{\prime} \\
& \times\left[1-3 \frac{\left(y_{m}-y_{n}\right)^{2}}{R_{m n}^{2}\left(z, z^{\prime}\right)}\right] \frac{\left[x_{m}(z)-x_{n}\left(z^{\prime}\right)\right]^{2}}{R_{m n}^{3}\left(z, z^{\prime}\right)}, \\
& E^{S E}=\frac{\mu b^{2}}{16 \pi} \sum_{m, n} \iint d z d z^{\prime} \frac{z-z^{\prime}}{R_{m n}^{3}\left(z, z^{\prime}\right)} x_{m}(z) \partial_{z^{\prime}} x_{n}\left(z^{\prime}\right) .
\end{aligned}
$$

As we did for the rigid line case, we can also express this elastic energy in Fourier space in order to diagonalize the interaction matrix and to obtain the wave-vector dependence of the interaction kernel between the different deformation modes. The detailed calculation is rather lengthy, so we merely indicate the procedure followed and the final results obtained. We evaluate separately the energy contribution due to the self-interaction between the constituent segments of each individual dislocation line, i.e., $n=m$, which we denote by $E_{0}$, and the energy contributions due to the interaction of dislocation segments lying on different lines, i.e., $n \neq m$, which we refer to as $E_{1}$. Proceeding this way, we find that the total energy is $E=E_{0}^{E E}+E_{0}^{E S}+E_{1}^{E E}+E_{1}^{E S}$. We express the dislocation displacements in terms of their Fourier modes, 


$$
x_{m}(z)=\int_{B Z} \frac{d k}{2 \pi} \int \frac{d q}{2 \pi} e^{-i k D m} e^{-i q z} x(k, q)
$$

and evaluate the self-interaction contributions for long wavelength deformations $q a \ll 1$ where $a$ is a short-distance cutoff introduced to preclude the interaction of a line segment with itself. The result can be written as

$$
\begin{aligned}
E_{0}^{E E}= & \frac{\mu b^{2}}{16 \pi(1-\nu)} \int_{B Z} \frac{d k}{2 \pi} \int \frac{d q}{2 \pi} \frac{1}{D} \\
& \times\left[2\left(\gamma-\frac{3}{2}+\ln a|q|\right) q^{2}-\frac{a^{2}}{12} q^{4}\right] x(k, q) x(-k,-q),
\end{aligned}
$$

$$
\begin{aligned}
E_{0}^{S E}= & \frac{\mu b^{2}}{8 \pi} \int_{B Z} \frac{d k}{2 \pi} \int \frac{d q}{2 \pi} \frac{1}{D}\left[2(-\gamma-\ln a|q|) q^{2}\right. \\
& \left.+\frac{a^{2}}{2} q^{4}\right] x(k, q) x(-k,-q),
\end{aligned}
$$

where $\gamma$ is the Euler constant. We find a quadratic wavevector dependence typical of a local interaction kernel but modified by logarithmic corrections. This a well-known result for isolated dislocation lines, as well as for similar singularities such as vortex lines in high-temperature superconductors. ${ }^{38}$

The energy contributions due to interactions between segments of different dislocation lines $(n \neq m)$ in the LAGB can be expressed as

$$
\begin{gathered}
E_{1}^{E E}=\frac{\mu b^{2}}{16 \pi(1-\nu)} \int_{B Z} \frac{d k}{2 \pi} \int \frac{d q}{2 \pi} \frac{1}{D}\left[2\left(\gamma+\ln \frac{D|q|}{4 \pi}\right) k^{2}+\frac{2 \pi}{D} \frac{k^{2}}{\left(k^{2}+q^{2}\right)^{1 / 2}}+\frac{D^{2}}{2 \pi^{2}} \zeta(3) k^{2} q^{2}\right] x(k, q) x(-k,-q), \\
E_{1}^{S E}=\frac{\mu b^{2}}{16 \pi} \int_{B Z} \frac{d k}{2 \pi} \int \frac{d q}{2 \pi} \frac{1}{D}\left[2\left(\gamma+\ln \frac{D|q|}{4 \pi}\right) q^{2}+\frac{2 \pi}{D} \frac{q^{2}}{\left(k^{2}+q^{2}\right)^{1 / 2}}+\frac{D^{2}}{2 \pi^{2}} \zeta(3) q^{4}\right] x(k, q) x(-k,-q),
\end{gathered}
$$

where $\zeta(x)$ is the Riemann zeta function. Naturally, the interaction kernel between the deformation modes for the threedimensional grain boundary case depends explicitly on both the $y$ and $z$ components of the wave vector in an intricate manner. Nevertheless, as in the two-dimensional case, for long wavelength deformations the leading term of the interaction kernel is essentially linear in the wave vector, which manifests the nonlocality of the interactions.

Finally, we consider the case of a pileup lying on the $x$ $=0$ plane. Again, we assume small perturbations of the dislocations from their equilibrium positions, $y_{m}-y_{n} \rightarrow\left[y_{m}\right.$ $\left.+\delta y_{m}(z)\right]-\left[y_{n}+\delta y_{n}\left(z^{\prime}\right)\right]$, where the displacements now depend on the $z$ coordinate of the infinitesimal line segment considered. Expanding up to first order in $\left[\delta y_{m}(z)-\delta y_{n}\left(z^{\prime}\right)\right]$, we evaluate the resulting Peach-Koehler glide forces and the corresponding elastic energy. As in the two-dimensional case, the result is equivalent to the one computed for the LAGB, provided that we replace $x_{n}(z)$ by $\delta y_{n}(z)$ in Eqs. (17) and (20). Thus also in this case we find wave-vector dependent interaction kernels whose leading terms (for long wavelength deformations) grow either quadratically in the wave vector (with logarithmic corrections) for self-interactions of the same dislocation line, or linearly in the case of interactions between different lines. Thus we may conclude that this particular form of the elastic kernels is characteristic of the long-range interactions between different dislocations. As we will see in the following, these long-range elastic properties have significant consequences for the analysis of the depinning transition of dislocation assemblies.

\section{DISORDER: DEPINNING TRANSITION}

Distortions in a LAGB or a pileup arise from interactions of the dislocations with various kinds of impurities such as solute atoms, precipitates, or other immobile defects. The interactions between individual dislocations and impurities have been computed and are reported in the literature. For the purpose of this paper, we will consider quenched disorder created by a random distribution of immobile impurities with concentration $c$ which interact with dislocations via a force $f_{p}(r)=f_{0} g\left(r / \xi_{p}\right)$, where $f_{0}$ is the pinning strength, $\xi_{p}$ is the interaction range, and $r$ is the distance between the impurity and the dislocation. The detailed shape $g(x)$ of the individual pinning force is inessential for most purposes.

The morphology and dynamics of a pileup or a LAGB result from a complicated interplay between elasticity and disorder. Pileup and LAGB are examples of the general problem of the depinning of elastic manifolds in random media, which has been extensively studied in the past. ${ }^{14}$ In the elastic approximation, the dynamics of the dislocation arrays follows:

$$
\chi \frac{\partial u}{\partial t}=\int d^{d} x^{\prime} K\left(x-x^{\prime}\right)\left[u\left(x^{\prime}\right)-u(x)\right]+b \sigma+\eta(x, u),
$$

where $\chi$ is a damping constant, $\sigma$ is the applied stress, $\eta(x, u)$ describes the effect of the pinning centers, and the elastic interaction kernel $K$, computed in the preceding section, scales as $|k|$ in Fourier space. In the following we will 
discuss how the main theoretical approaches to the depinning transition can be applied to the problem at hand.

\section{A. Collective pinning theory: Weak pinning}

Collective pinning theory describes the behavior of the LAGB in the limit of weak disorder, when pinning is due to the fluctuations of the random forces. The key concept is the introduction of a characteristic length $L_{c}$ above which pinning becomes effective (or energetically advantageous) and consequently the LAGB is distorted. The collective pinning length can be evaluated, for instance, by balancing the elastic energy cost and the pinning energy gain associated with a small displacement of a region of linear size $L$. On scales below $L$, the dislocations remain essentially undeformed and, hence, the fluctuations in potential energy follow Poissonian statistics. The effective concentration of the pinning defects along the LAGB is given by

$$
\begin{aligned}
& \bar{c}_{\mathrm{eff}}=\left\{\begin{array} { l } 
{ \overline { c } , \xi _ { p } > D } \\
{ \overline { c } \frac { \xi _ { p } } { D } , \xi _ { p } < D }
\end{array} \left(\begin{array}{l}
(2 \mathrm{D}), \\
c_{\mathrm{eff}}=\left\{\begin{array}{l}
c, \xi_{p}>D \\
c \frac{\xi_{p}}{D}, \xi_{p}<D
\end{array}\right. \text { (3D). }
\end{array}\right.\right.
\end{aligned}
$$

The first expression refers to pinning by columnar defects of areal concentration $\bar{c}$ in $d=2$, and the second to pinning by localized defects of volume concentration $c$ in $d=3$. In $d$ $=2$, the characteristic energy of a section of a LAGB of size $L$ displaced by an amount of the order of $u$ can be written as

$$
\bar{E}=\frac{\mu b^{2} u^{2}}{D^{2}}-\bar{f}_{0} \xi_{p} \sqrt{\bar{c}_{\text {eff }} L u} .
$$

Here both $\bar{E}$ and $\bar{f}_{0}$ are defined as quantities per unit length. In the case of a thin film of thickness $h$, one can obtain their three-dimensional counterparts just as $E=h \bar{E}$ and $f_{0}=h \bar{f}_{0}$. Note the scale independence of the nonlocal expression of the elastic energy $\mu b^{2} u^{2} / D^{2}$ in contrast to what would be this energy in the local approximation $\mu b^{2} u^{2} / D L$. Essentially the same expression holds for the pileup. Balancing elastic and pinning contributions and imposing that the displacement is of the order of the pinning range $u \sim \xi_{p}$, one readily obtains $L_{c}=\left(\mu^{2} b^{4} \xi_{\mathrm{p}}\right) /\left(D^{4} \bar{f}_{0}^{2} \bar{c}_{\text {eff }}\right)$. The LAGB is depinned when the work done by the external stress in moving a segment of length $L_{c}$ over the distance $\xi_{p}$ exceeds the characteristic pinning energy $\bar{E}\left(L_{c}\right)$ of this segment. Equating $\bar{E}\left(L_{c}\right)$ $=\sigma_{c} b L_{c} \xi_{p} / D$, for the case above the result is given by $\sigma_{c} b$ $=\left(\bar{c}_{\text {eff }} \bar{f}_{0}^{2} D^{3}\right) /\left(\mu b^{2}\right)$.

A similar calculation in $d=3$ is more subtle, since the elastic and the pinning energies scale with the same power of $L$ and thus cancel in the simple dimensional approach discussed above. As we will discuss in the following section, this reflects the fact that $d=3$ is the upper critical dimension for the transition. To obtain $L_{c}$ in this case, one should perform a perturbation expansion in the disorder, as discussed in
Ref. 12 in the context of the flux line lattice. One essentially computes the typical displacement $u$ for a system of size $|\mathbf{r}|=L$, which for a LAGB is given by

$$
\begin{aligned}
\left\langle|u(\mathbf{r})-u(0)|^{2}\right\rangle= & \int \frac{d^{2} k}{(2 \pi)^{2}} \int \frac{d^{2} k^{\prime}}{(2 \pi)^{2}}(1-\cos \mathbf{k} \cdot \mathbf{r}) \\
& \times G(\mathbf{k}) G\left(\mathbf{k}^{\prime}\right) F(\mathbf{k}) F\left(\mathbf{k}^{\prime}\right),
\end{aligned}
$$

where $G(\mathbf{k})$ is the Green function associated with the elastic kernel determined in the preceding section, and $F(\mathbf{k})$ is the pinning force density. In the spirit of collective pinning theory $\left\langle F(\mathbf{k}) F\left(\mathbf{k}^{\prime}\right)\right\rangle=W \delta^{(2)}\left(\mathbf{k}+\mathbf{k}^{\prime}\right)$ with $W=\left(f_{0} \sqrt{c_{\text {eff }} \xi_{p}}\right)^{2}$. The explicit calculation leads to the characteristic displacement

$$
u(L) \simeq f_{0} \sqrt{c_{\mathrm{eff}} \xi_{p}} \frac{D^{2}}{\mu b^{2}} \ln ^{1 / 2} \frac{L}{D} .
$$

This expression can then be inverted, imposing $u \sim \xi_{p}$, to obtain

$$
L_{c}=D \exp \left[\frac{\xi_{p}}{c_{\text {eff }}}\left(\frac{\mu b^{2}}{f_{0} D^{2}}\right)^{2}\right] .
$$

The depinning stress can then be obtained as in $d=2$ and is given by $\sigma_{c} b=\left(\mu b^{2} \xi_{p}\right) /\left(D L_{c}\right)$. Again these results generalize directly to the pileup case. It is, however, important to note that they refer to the continuum limit, when one can neglect the discrete nature of the dislocation system. To be consistent with this assumption, one should have $L_{c} \gg D$.

\section{B. Strong pinning: Friedel statistics}

Collective pinning is due to a statistical superposition of the forces created by many obstacles. In the limit of strong and/or diluted pinning centers, however, the characteristic bulge of width $\xi_{p}$ and extension $L_{c}$ as envisaged in the preceding section may not interact with enough pinning centers for this viewpoint to be valid. Simple estimates for the boundaries of the collective pinning regime are given by the inequalities $L_{c} \xi_{p} \geqslant 1 / \bar{c}_{\text {eff }}$ and $L_{c}^{2} \xi_{p} \geqslant 1 / c_{\text {eff }}$ for the $d=2$ and $d=3$ cases discussed above, respectively.

In the regime of strong pinning, dislocations are pinned by individual obstacles. The spacing of obstacles along the dislocation and the depinning stress can be obtained by an argument which was, in the context of single dislocations, developed by Friedel. The basic idea is to consider the behavior of a dislocation segment as it depins from a pair of strong obstacles. The length of the segment is $L$, and it forms a bulge of width $u$. If the dislocation segment overcomes one of the pins it will travel by an amount which is, again, of the order of $u$ and, hence, sweep an area of the order of $L u$. Now we can estimate the depinning threshold by requiring that during this process the freed dislocation segment encounters, on average, precisely one new obstacle. In other words, precisely at the point of depinning the dislocation starts to move through a sequence of statistically equivalent configurations. For a dislocation this leads to the condition $L u \simeq 1 /\left(c \xi_{p}\right) . L$ and $u$ can be related by equating the work done by the external stress $\sigma$ in bulging out the dislocation to the concomitant elastic energy increase, $\Gamma u^{2} / L=\sigma b u L$, where $\Gamma$ is a con- 
TABLE I. Overview of pinning stresses and pinning lengths obtained from different models and their physical realizations.

\begin{tabular}{|c|c|c|c|c|c|}
\hline $\begin{array}{l}\text { Model } \\
\text { dimension }\end{array}$ & $\begin{array}{l}\text { Type of } \\
\text { elasticity }\end{array}$ & $\begin{array}{l}\text { Type of } \\
\text { pinning }\end{array}$ & Pinning length & Critical stress & Physical realization \\
\hline $2 \mathrm{D}$ & Local & Weak & $L_{c}=\left(\Gamma_{0}^{2} \xi_{p} / \bar{f}_{0}^{2} \bar{c}_{\text {eff }}\right)^{1 / 3}$ & $\sigma_{c} b=\left(D^{3} \bar{f}_{0}^{4} \xi_{p} / \Gamma_{0}\right)^{1 / 3}$ & Isolated dislocation \\
\hline $2 \mathrm{D}$ & Local & Strong & $L_{f}=\left(\Gamma_{0} / \bar{f}_{0} \bar{c}_{\mathrm{eff}}\right)^{1 / 2}$ & $\sigma_{c} b=\left(D^{2} \bar{f}_{0}^{3} \bar{c}_{\mathrm{eff}} / \Gamma_{0}\right)^{1 / 2}$ & Isolated dislocation \\
\hline $2 \mathrm{D}$ & Nonlocal & weak & $L_{c}=\Gamma_{0}^{2} \xi_{p} / D^{2} \bar{f}_{0}^{2} \bar{c}_{\mathrm{eff}}$ & $\sigma_{c} b=D^{2} \bar{f}_{0}^{2} \bar{c}_{\mathrm{eff}} / \Gamma_{0}$ & $\begin{array}{l}\text { Dislocation array, } \\
\text { rigid dislocations }\end{array}$ \\
\hline $2 \mathrm{D}$ & Nonlocal & Strong & $L_{f}=\Gamma_{0} / D \bar{f}_{0} \bar{c}_{\mathrm{eff}}$ & $\sigma_{c} b=D^{2} \bar{f}_{0}^{2} \bar{c}_{\mathrm{eff}} / \Gamma_{0}$ & $\begin{array}{l}\text { Dislocation array, } \\
\text { rigid dislocations }\end{array}$ \\
\hline $3 \mathrm{D}$ & Local & Weak & $L_{c}=\left(\Gamma_{0}^{2} \xi_{p} / f_{0}^{2} c_{\mathrm{eff}}\right)^{1 / 2}$ & $\sigma_{c} b=D f_{0}^{2} c_{\mathrm{eff}} / \Gamma_{0}$ & Elastic sheet \\
\hline $3 \mathrm{D}$ & Local & Strong & $L_{f}=\left(\Gamma_{0} / f_{0} c_{\mathrm{eff}}\right)^{1 / 2}$ & $\sigma_{c} b=D f_{0}^{2} c_{\mathrm{eff}} / \Gamma_{0}$ & Elastic sheet \\
\hline $3 \mathrm{D}$ & Nonlocal & Weak & $L_{c}=D \exp \left[\Gamma_{0}^{2} \xi_{p} / D^{2} f_{0}^{2} c_{\text {eff }}\right]$ & $\sigma_{c} b=\Gamma_{0} \xi_{p} D \exp \left[-\Gamma_{0}^{2} \xi_{p} D^{2} f_{0}^{2} c_{\mathrm{eff}}\right]$ & $\begin{array}{l}\text { Dislocation array, } \\
\text { flexible dislocations }\end{array}$ \\
\hline $3 \mathrm{D}$ & Nonlocal & Strong & $L_{f}=\Gamma_{0} / D f_{0} c_{\mathrm{eff}}$ & $\sigma_{c} b=D^{3} f_{0}^{3} c_{\mathrm{eff}}^{2} / \Gamma_{0}^{2}$ & $\begin{array}{l}\text { Dislocation array, } \\
\text { flexible dislocations }\end{array}$ \\
\hline
\end{tabular}

stant line tension. Finally, the depinning force can be obtained by comparing the external force $b \sigma L$ with the pinning force $f_{0}$. Solving these three equations, one obtains the Friedel length $L_{f} \simeq\left(\Gamma / c \xi_{p} f_{0}\right)^{1 / 2}$ and the depinning stress $\sigma_{c} b \simeq\left(c \xi_{p} f_{0}^{3} / \Gamma\right)^{1 / 2}$.

This argument can be generalized in a straightforward manner to the case of dislocation arrays. Let us first consider the depinning of a two-dimensional LAGB as discussed above in the weak pinning limit: In this case, the Friedel condition reads $L u \simeq 1 / \bar{c}_{\text {eff }}$; the elastic energy per unit length of a bulge of width $u$ and extension $L$ is $\mu b^{2} u^{2} / D^{2}$ which must equal the work per unit length $\sigma b L u / D$; and the force balance (again per unit length) is $\sigma_{c} b L / D=\bar{f}_{0}$. Combining these relations we find that the Friedel length and the depinning stress are

$$
L_{f} \simeq \frac{\mu b^{2}}{D^{2} \bar{c}_{\mathrm{eff}} \bar{f}_{0}}, \quad \sigma_{c} b \simeq \frac{\bar{c}_{\mathrm{eff}} \bar{f}_{0}^{2} D^{3}}{\mu b^{2}}
$$

In $3 \mathrm{D}$ the Friedel condition is $L^{2} u \simeq 1 / c_{\text {eff }}$, the energy balance reads $\mu b^{2} u^{2} L / D^{2}=\sigma b L^{2} u / D$, and the force balance is $\sigma_{c} b L^{2} / D=f_{0}$. This yields

$$
L_{f} \simeq \frac{\mu b^{2}}{D^{2} c_{\mathrm{eff}} f_{0}}, \quad \sigma_{c} b \simeq \frac{c_{\mathrm{eff}}^{2} f_{0}^{3} D^{5}}{\mu^{2} b^{4}} \quad \text { (3D) } .
$$

Table I presents a compilation of results for the weak and strong pinning cases in two and three dimensions. For comparison we have also included results obtained under the assumption that the elastic behavior of the grain boundary can, in local elasticity approximation, be described by a scaleindependent surface energy $\Gamma_{0} \sim \mu b^{2} / D$.

\section{DYNAMICS: CRITICAL SCALING NEAR THE DEPINNING THRESHOLD}

Second-order phase transitions can be described by scaling laws and critical exponents and the depinning transition is no exception. In the system discussed here, the control parameter is the applied stress, so that scaling laws depend on the distance $\sigma-\sigma_{c}$ from the critical point. In particular, as the system approaches the transition, the correlation length diverges as $\xi \sim\left(\sigma-\sigma_{c}\right)^{-\nu}$. Similarly, one can define a characteristic correlation time $t^{*}$, related to the correlation length as $t^{*} \sim \xi^{z}$. The average dislocation velocity reaches a steady value, scaling as $v \sim\left(\sigma-\sigma_{c}\right)^{\beta}$, above the transition, and vanishes below. Before the steady state the average velocity decays as a power law $t^{-\theta}$, for times $t<t^{*}$. Furthermore, the Orowan relation, which relates the rate of plastic deformation $\dot{\gamma}$ to the density $\rho$ and average velocity $v$ of moving dislocations in a crystal, implies that similar scaling laws should hold for the strain rate $\dot{\gamma} \equiv b \rho v$. In this respect, it is tempting to establish a relationship between the dynamical behavior of dislocation systems and the creep laws observed in plastically deforming crystals, i.e., the crossover between primary (power law) to secondary (linear) creep.

Scaling exponents also characterize the morphology of the dislocation arrangement, which exhibits roughening close to the depinning transition. The roughness can be quantified measuring the average displacement correlations $C\left(x-x^{\prime}\right)$ $=\left\langle\left[u(x)-u\left(x^{\prime}\right)\right]^{2}\right\rangle$. At the transition in the steady state, we expect a self-affine scaling $C(x) \sim x^{2 \zeta}$, where $\zeta$ is the roughness exponent while the transient behavior is described by a scaling form of the type $C(x, t)=t^{\beta_{t}} f\left(x / t^{1 / z}\right)$. As in ordinary critical phenomena, only a fraction of the scaling exponents are independent. For instance, one can easily derive the relations $\beta_{t}=z / \zeta$ and $\theta=\beta /(\nu z)$. 


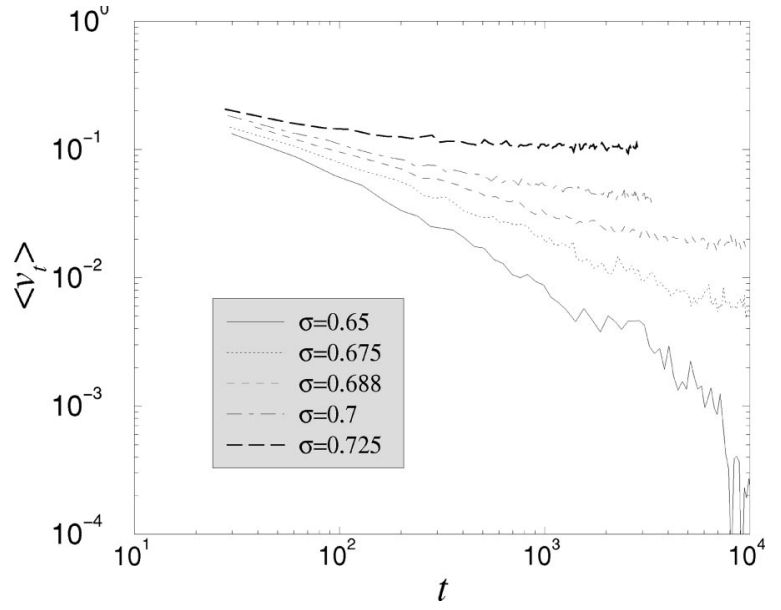

FIG. 4. The decay of the average pileup velocities as a function of the applied stress $\sigma$. For $\sigma>\sigma_{c} \simeq 0.675$ the velocity reaches a steady value and decays to zero otherwise.

In general, it has been shown that in the depinning problem there are only two independent exponents that have been computed using the renormalization-group. To connect our problem to previously obtained results, we notice that the effective elastic energy of the pileup and LAGB scales as $|q|$ in Fourier space, as in the problems of contact line $e^{49}$ and planar crack depinning. ${ }^{50}$ We can thus directly apply to our case the results obtained for a contact line with long-range elastic energy. ${ }^{14,26}$ The renormalization group analysis predicts that $d_{c}=3$ is the upper critical dimension, above which fluctuations are suppressed. Thus for $d>d_{c}$ there is no roughening (i.e., $\zeta=0$ ) and the other exponents can be computed in the mean-field approximation, yielding $\beta=z=\nu=1$. These results are valid in the physically interesting dimension $d=3$ apart from additional logarithmic corrections. For $d<3$, a renormalization-group expansion in $\epsilon=3-d$ has been performed to compute the exponents which at first order in $\epsilon$ are given by $\beta=7 / 9, \nu=3 / 2, \zeta=1 / 3$, and $z=7 / 9 .{ }^{26}$ Using the scaling relation $\theta=\beta /(\nu z)$ one obtains $\theta=2 / 3$, which coincides with the exponent of the so-called Andrade creep law, observed in the creep deformation of several materials. ${ }^{13,43}$

\section{A. Two-dimensional pileup: Numerical simulations}

Our theoretical results have been obtained assuming small perturbations of a regular dislocation arrangement. In order to test the validity of these results in the general case where arbitrary dislocation positions are permitted, we have performed a series of numerical simulations of the dynamics of a two-dimensional pileup. This corresponds to an effective one-dimensional model in which $N$ interacting point dislocations move along a line in presence of quenched disorder. For simplicity, we consider periodic boundary conditions, so that in absence of disorder the equilibrium configuration is an equally spaced pileup. To test the dependence on the system size, we change the dislocation number $N$ and the system size $L$, keeping the dislocation spacing $D=L / N$ constant.

The equation of motion for the dislocation $i$ in the pileup is given by

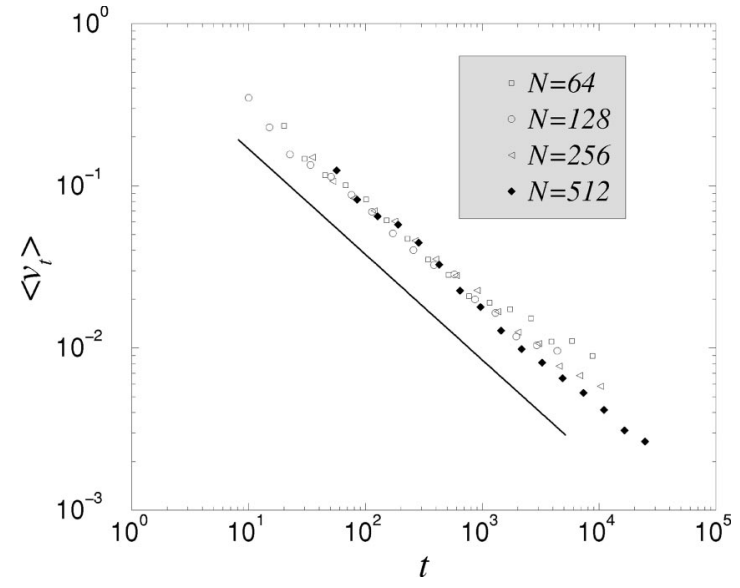

FIG. 5. The decay of the velocity at $\sigma>\sigma_{c} \simeq 0.675$ for different values of $N$. As $N$ increases, the power-law scaling region extends. The line has a slope of $\theta=0.65$.

$$
\chi \frac{d x_{i}}{d t}=\mu b^{2} \sum_{j \neq i} \frac{1}{\left|x_{i}-x_{j}\right|}+b \sigma+\sum_{P} f_{p}\left(x_{i}-X_{P}\right)
$$

where $\chi$ is an effective viscosity and $\sigma$ is the applied stress. The pinning centers are placed at randomly chosen positions $X_{P}$ (with $P=1, \ldots, N_{P}$ ) and exert an attractive force on the dislocations,

$$
f_{p}(x)=-f_{0} \frac{x}{\xi_{p}} e^{-\left(x / \xi_{p}\right)^{2}}
$$

In order to correctly take into account the effect of periodic boundary conditions, the interactions between dislocations are summed over the images. In one dimension the sum can be performed exactly and $1 /|x|$ in Eq. (29) is replaced by

$$
\sum_{k=-\infty}^{\infty} \frac{1}{x+k L}=\frac{\pi}{L \tan (\pi x / L)}
$$

The equation of motion [Eq. (29)] is integrated numerically using a Runge-Kutta algorithm for different values of the applied stress. We take as initial condition a perfectly ordered pileup, with equally spaced dislocations. For the simulations reported here, we first considered $N$ $=64,128,256,512$ dislocations with a spacing $D=16$ and average pinning center spacing $d_{p} \equiv L / N_{p}=2$. The units of time, space, and forces are chosen so that $\mu b^{2}=1, \chi=1$, and $b=1$, and we set $f_{0}=1$ and $\xi_{p}=1$.

In Fig. 4 we report the time decay of the average pileup velocity for different values of the applied stress. For large stress values, $\sigma>\sigma_{c} \simeq 0.675$, the initial power-law decay is followed by a plateau, while the velocity decays to zero otherwise. This allows to identify the depinning point as $\sigma_{c}$ $\simeq 0.675$. This is confirmed by the finite size analysis shown in Fig. 5, indicating that for $\sigma_{c}=0.675$ the power law extends further as the system size is increased. The exponent of the power-law scaling $\theta \simeq 0.65$ is in good agreement with the theoretical expectations. 


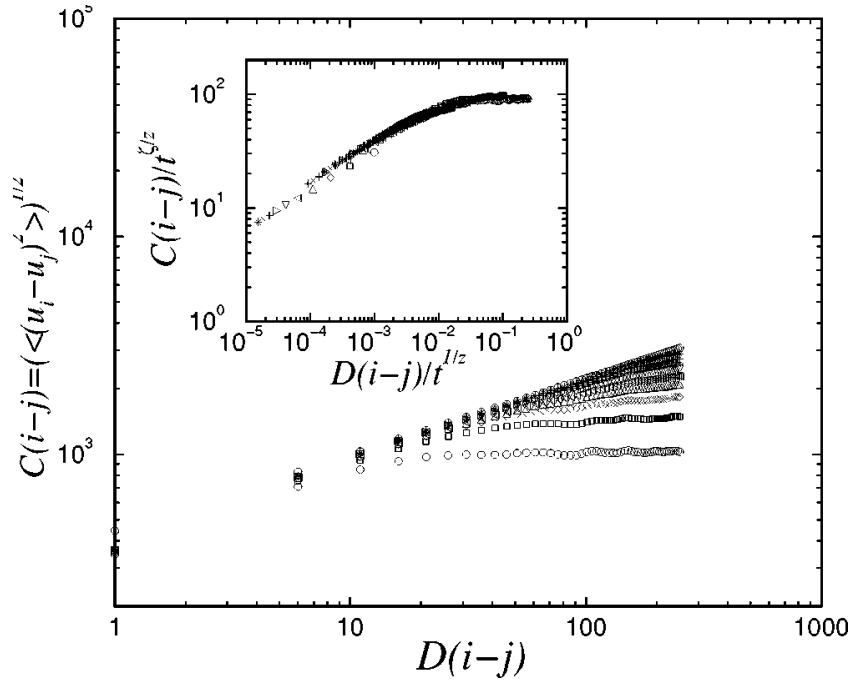

FIG. 6. The growth of the correlation function at the depinning transition at different times. The data collapse in the inset allows to estimate the roughness exponent $\zeta=0.35$ and the dynamic exponent $z=0.9$.

Moreover, in order to characterize the growth of correlations at the critical point, we compute the displacement correlation function $C(i-j, t)=\left\{\left\langle\left[u_{i}(t)-u_{j}(t)\right]^{2}\right\rangle\right\}^{1 / 2}$ at different times $t$ for $\sigma=\sigma_{c}$ (see Fig. 6). The curves can be collapsed using the scaling form $C(x, t)=t^{\zeta / z} f\left(x / t^{1 / z}\right)$ with $\zeta=0.35$ and $z=0.9$ (see the inset of Fig. 6). To confirm this result we have also computed the evolution of the power spectrum $P(k, t)$ $=\int d x C(x) \exp (i k x)$ (Fig. 7). These curves can also be collapsed as $P(k, t)=t^{(2 \zeta+1) / z} g\left(k t^{1 / z}\right)$ with the same exponent values as the correlation function.

In summary, all the exponents determined from the simulations are in good agreement with the renormalization-group predictions and with previous simulations based directly on the elastic approximation, confirming the validity of the elastic theory for the pileup.

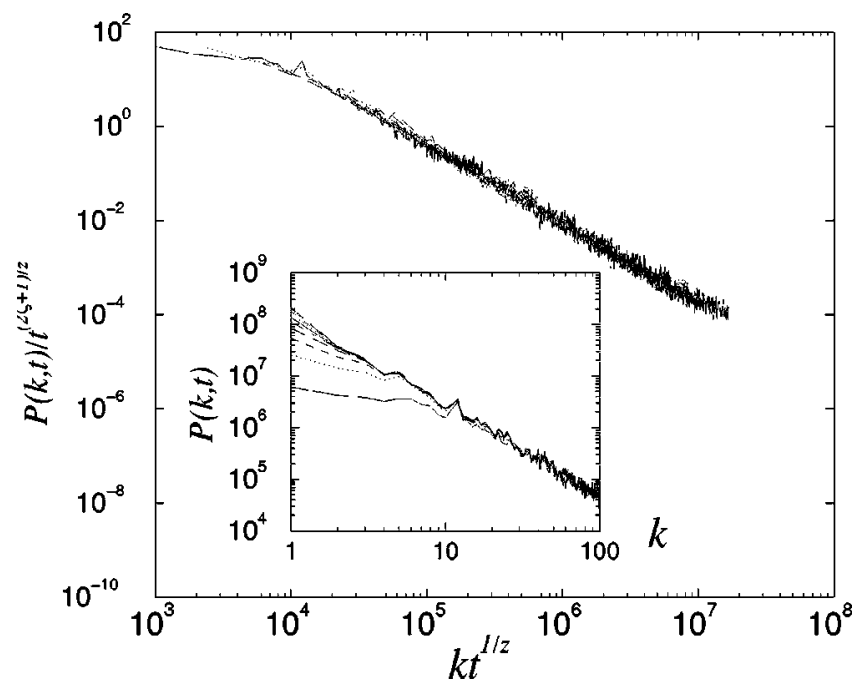

FIG. 7. The power spectrum of the pileup at the depinning transition. The data collapse is consistent with the scaling of the correlation function.

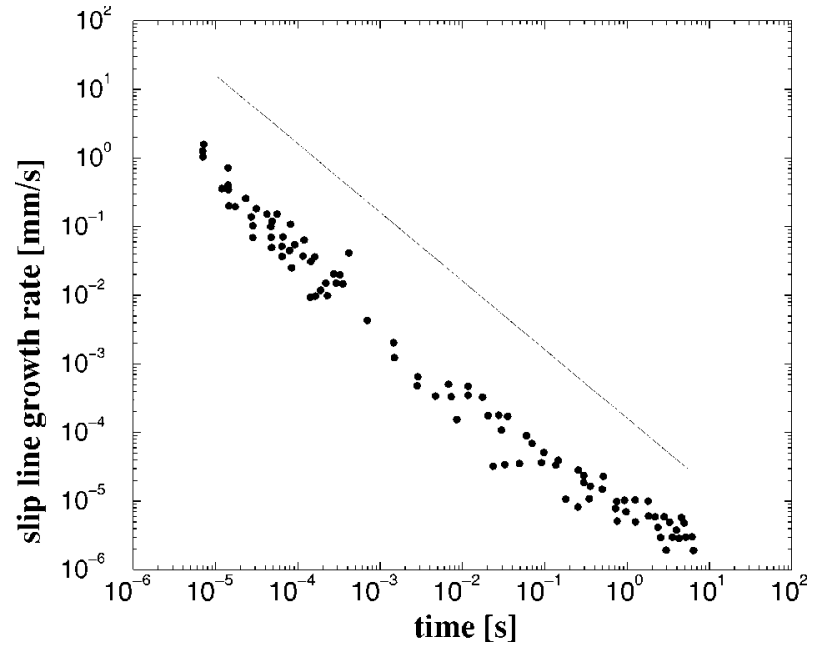

FIG. 8. Growth rate of slip steps on the surface of $\mathrm{Cu}-30$ at \% $\mathrm{Zn}$ deformed at room temperature as a function of the time passed after growth has started; after Ref. 9. The line is a power law with exponent $\theta=1$.

\section{B. Three-dimensional pileup: Relaxation of slip-band growth rates}

In the case of flexible dislocation lines, we expect the depinning transition of a planar dislocation array to be governed by mean-field exponents. A detailed discussion of this case has been given elsewhere in the context of domain-wall depinning. ${ }^{16}$ The mean-field exponents are $\beta=z=\nu=1$; for a theoretical derivation and confirmation by numerical simulations the reader is referred to the previous work. Here, we only point out a possible experimental check in the context of planar dislocation arrangements.

Direct experimental observation of the dynamics of planar dislocation arrays may be possible in certain alloys exhibiting so-called planar slip where dislocations form huge pileups (see Fig. 1). The motion of these planar dislocation groups goes along with the formation of large slip steps along the traces where the slip plane of the pileup intersects the surface of the metal specimen. For a moving pileup consisting of roughly equally spaced dislocations, the slip step growth rate is proportional to the dislocation velocity. Since often only a small number of slip steps are growing at a time, ${ }^{51}$ one may attempt to relate the observed time dependence of slip step growth to the velocity relaxation of a single pileup.

Figure 8 shows experimental data with rates of slip step growth as a function of the time after growth has started. ${ }^{9}$ The double-logarithmic plot indicates relaxation of the growth rate (the dislocation velocity) according to $v \propto t^{-\theta}$ with a characteristic exponent $\theta=1 \pm 0.1$ over six decades. On the other hand, for the depinning transition of a planar dislocation array in $3 \mathrm{D}$ we expect according to the scaling relation $\theta=\beta /(\nu z)$ the value $\theta=1$. The apparent length of the scaling regime indicates that driving of the dislocation arrays occurs at stresses very close to the critical one. This is in line with the general observation that dislocation arrangements in slowly deforming crystals (where "slow" covers the entire range of strain rates used in typical experiments ${ }^{52}$ ) are in a close-to-critical state. ${ }^{52,53}$ 


\section{CONCLUSIONS}

We have investigated the depinning transition of planar dislocation arrays such as small-angle grain boundaries or dislocation pileups. Contrary to the case of isolated dislocations, the elastic interactions between dislocation line segments in such arrays are of long-range nature and, hence, cannot be described within a line- or surface-tension approximation. The pinning of planar dislocation arrays has been investigated both in the weak and strong pinning limits using collective pinning theory and Friedel statistics, respectively.

In certain situations, our results may have some implications for grain growth limited by grain boundary pinning. ${ }^{40,41}$ For instance, in Ref. 42 the derivation of the dependence of the average grain size $R$ on the impurity concentration $c$ involved a Friedel-type estimate of the depinning stress for a grain boundary. The calculation was made in a local elasticity (grain boundary energy) approximation, neglecting longrange stresses. If these were included the result would change dramatically as discussed in Sec. III B. However, the present results apply only when grain boundary mobility is governed by glide of the GB dislocations. In the general case where grain boundary motion is controlled by diffusional rearrangements (glide-climb of the grain boundary dislocations) long-range stresses need not occur and local elasticity approximations may retain their validity.

Long-range elastic interactions also govern the dynamics of planar dislocation arrays at the depinning threshold. In two dimensions, computer simulations and theoretical arguments suggest that the dynamics falls into the same class as contact-line depinning, while in three dimensions the dynamical behavior can be described by mean-field exponents. In particular, we have demonstrated that the mean-field pre- diction for the velocity relaxation of a planar dislocation array (pileup) is consistent with experimental observations of the time-dependent growth of slip bands in alloys exhibiting planar slip.

The dislocation arrangements discussed in the present study have a simple, quasiplanar geometry in which only dislocations of one sign are present and only small perturbations of the planar arrangement of the dislocations are permitted. Because of this particular geometry, the dislocation assemblies behave like two- or three- dimensional longrange elastic objects. The situation is much more complicated when dislocations of different types and directions of motion have to be considered. In such situations, there is still a transition between a stationary and a moving state of the dislocation assembly ("yielding transition"). ${ }^{43}$ However, in general dislocation assemblies the existence of metastable stationary states does not depend on the presence of quenched disorder as in the present study. Rather, the interactions between dislocation lines of different type together with the dynamics constraints which tie the motion of the dislocation lines to their respective slip planes lead to the possibility of forming metastable jammed configurations even in the absence of any disorder. While the general scenario of dynamic nonequilibrium phase transitions applies to such systems, no ready-made theoretical framework is available and, hence, a theory of the yielding and dynamic behavior of general dislocation systems remains a formidable task for future investigations.

\section{ACKNOWLEDGMENTS}

This work was supported by an Italy-Spain Integrated Action. M.C.M. acknowledges financial support from the Ministerio de Ciencia y Tecnología (Spain).
${ }^{1}$ F. R. N. Nabarro, Proc. R. Soc. London, Ser. A 381, 285 (1982).

${ }^{2}$ E. H. Brandt, Phys. Rev. Lett. 56, 1381 (1986).

${ }^{3}$ L. B. Ioffe and V. M. Vinokur, J. Phys. C 20, 6149 (1987).

${ }^{4}$ J. G. Sevillano, E. Bouchaud, and L. P. Kubin, Scr. Metall. Mater. 25, 355 (1991).

${ }^{5}$ G. D’Anna, W. Benoit, and V. M. Vinokur, J. Appl. Phys. 82, 5983 (1997).

${ }^{6}$ S. Zapperi and M. Zaiser, Mater. Sci. Eng., A 309-310, 348 (2001).

${ }^{7}$ Dislocations in Solids, edited by F. R. N. Nabarro (NorthHolland, Amsterdam, 1979) Vol. 4.

${ }^{8}$ M. Z. Butt and P. Feltham, J. Mater. Sci. 28, 2557 (1993).

${ }^{9}$ H. Neuhäuser, Phys. Scr., T 49, 412 (1993).

${ }^{10}$ R. Labusch, Phys. Status Solidi 41, 659 (1970); Acta Metall. 20, 917 (1972).

${ }^{11}$ R. Labusch, Cryst. Lattice Defects 1, 1 (1969).

${ }^{12}$ A. I. Lar'kin and Yu. N. Ovchinnikov, J. Low Temp. Phys. 34, 409 (1979).

${ }^{13}$ J. Friedel, Dislocations (Pergamon Press, Oxford, 1967).

${ }^{14}$ M. Kardar, Phys. Rep. 301, 85 (1998).

${ }^{15}$ S. Lemerle, J. Ferré, C. Chappert, V. Mathet, T. Giamarchi, and P. Le Doussal, Phys. Rev. Lett. 80, 849 (1998).
${ }^{16}$ S. Zapperi, P. Cizeau, G. Durin, and H. E. Stanley, Phys. Rev. B 58, 6353 (1998).

${ }^{17}$ S. Bhattacharya and M. J. Higgins, Phys. Rev. Lett. 70, 2617 (1993).

${ }^{18}$ R. Surdeanu, R. J. Wijngaarden, E. Visser, J. M. Huijbregtse, J. H. Rector, B. Dam, and R. Griessen, Phys. Rev. Lett. 83, 2054 (1999).

${ }^{19}$ E. Schäffer, and P.-z. Wong, Phys. Rev. E 61, 5257 (2000).

${ }^{20}$ E. Rolley, C. Guthmann, R. Gombrowicz, and V. Repain, Phys. Rev. Lett. 80, 2865 (1998).

${ }^{21}$ E. Bouchaud, J. Phys.: Condens. Matter 9, 4319 (1997).

${ }^{22}$ J. Schmittbuhl and K. Måløy, Phys. Rev. Lett. 78, 3888 (1997).

${ }^{23}$ T. Nattermann, S. Stepanow, L. H. Tang, and H. Leschhorn, J. Phys. II 2, 1483 (1992).

${ }^{24}$ H. Leschhorn, T. Nattermann, S. Stepanow, and L. H. Tang, Ann. Phys. (Leipzig) 6, 1 (1997).

${ }^{25}$ O. Narayan and D. S. Fisher, Phys. Rev. B 48, 7030 (1993).

${ }^{26}$ D. Ertas and M. Kardar, Phys. Rev. E 49, R2532 (1994).

${ }^{27}$ P. Chauve, T. Giamarchi, and P. Le Doussal, Phys. Rev. B 62, 6241 (2000).

${ }^{28}$ P. Le Doussal, K. J. Wiese, and P. Chauve, Phys. Rev. B 66, 174201 (2002). 
${ }^{29}$ D. Cule and T. Hwa, Phys. Rev. Lett. 77, 278 (1996); Phys. Rev. B 57, 8235 (1998).

${ }^{30}$ F. Lacombe, S. Zapperi, and H. J. Herrmann, Phys. Rev. B 63, 104104 (2001).

${ }^{31}$ A. Rosso, A. K. Hartmann, and W. Krauth, Phys. Rev. E 67, 021602 (2003).

${ }^{32}$ J. Schmittbuhl, S. Roux, J. P. Villotte, and K. J. Maloy, Phys. Rev. Lett. 74, 1787 (1995).

${ }^{33}$ S. Ramanathan and D. Fisher, Phys. Rev. Lett. 79, 877 (1997); Phys. Rev. B 58, 6026 (1998).

${ }^{34}$ A. Tanguy, M. Gounelle, and S. Roux, Phys. Rev. E 58, 1577 (1998).

${ }^{35}$ A. Rosso and W. Krauth, Phys. Rev. E 65, 025101 (2002).

${ }^{36}$ A. J. E. Foreman, Philos. Mag. 15, 1011 (1967).

${ }^{37}$ G. De Wit and J. S. Koeheler, Phys. Rev. 116, 1113 (1959).

${ }^{38}$ G. Blatter, M. V. Feigelman, V. B. Geshkenbein, A. I. Larkin, and V. M. Vinokur, Rev. Mod. Phys. 66, 1125 (1994).

${ }^{39}$ M. C. Miguel and M. Kardar, Phys. Rev. B 56, 11903 (1997).

${ }^{40}$ C. S. Smith, Trans. Metall. Soc. AIME 175, 15 (1948).

${ }^{41}$ P. A. Manohar, M. Ferry, and T. Chandra, ISIJ Int. 38, 913 (1998).

${ }^{42}$ P. M. Hazzledine and R. D. J. Oldershaw, Philos. Mag. A 61, 579 (1990).
${ }^{43}$ M. C. Miguel, A. Vespignani, M. Zaiser, and S. Zapperi, Phys. Rev. Lett. 89, 165501 (2002).

${ }^{44}$ J. Plessing, Ph.D. thesis, University of Braunschweig, 1995.

${ }^{45}$ S. I. Zaitsev and E. M. Nadgorny, Fiz. Tverd. Tela (Leningrad) 21, 1392 (1979) [Sov. Phys. Solid State 21, 804 (1979)]; 21, 3349 (1979) [21, 1934 (1979)].

${ }^{46}$ J. P. Hirth and J. Lothe, Theory of Dislocations (McGraw-Hill, New York, 1968).

${ }^{47}$ An analogous expression was derived in S. T. Chui, Phys. Rev. B 28, 178 (1983).

${ }^{48}$ Because of the emergence of "corners" in the dislocation lines, this yields a rather poor description of the interaction between adjacent line segments. However, in the following we are mainly interested in the long-wavelength elasticity of the dislocation lines, which in this staircase approximation is captured exactly in the limit of infinitesimally small segment lengths.

${ }^{49}$ J. F. Joanny and P. G. de Gennes, J. Chem. Phys. 81, 552 (1984).

${ }^{50}$ H. Gao and J. R. Rice, ASME J. Appl. Mech. 56, 828 (1989).

${ }^{51}$ H. Neuhäuser, in Dislocations in Solids, edited by F. R. N. Nabarro (North-Holland, Amsterdam, 1983) Vol. 6, p. 319.

${ }^{52}$ M. Zaiser, Mater. Sci. Eng., A 309/310, 304 (2001).

${ }^{53}$ M.-C. Miguel, A. Vespignani, S. Zapperi, J. Weiss, and J. R. Grasso, Nature (London) 410, 667 (2001). 